\title{
Slices Como Serviço Sobre um Centro de Dados Itinerante Aplicado ao Cenário Amazônico
}

\author{
Josiane Ribeiro ${ }^{1}$, Lucas Borges ${ }^{1}$, Paulo Senna ${ }^{1}$, Billy Pinheiro ${ }^{1}$, Antônio Abelém ${ }^{1}$ \\ ${ }^{1}$ Grupo de Pesquisa em Redes de Computadores e Comunicação Multimídia (GERCOM) \\ Universidade Federal do Pará (UFPA) \\ Caixa Postal 470 - 66075-110 - Belém - PA - Brasil \\ \{josianeribeiro796, borgeslucas873, paulosena99\}@gmail.com
}

$\{$ billy, abelem\}@ufpa.br

\begin{abstract}
Virtualization and resource slicing are becoming key approaches to automating, streamlining and cost-cutting the cloud configuration process, enabling the provision of agile and dynamic services. This paper presents the Itinerant Data Center (IDC), with the objective of bringing essential services to regions with or without infrastructure, using the NECOS platform, to provide a sustainable, low-cost and with low energy consumption, which uses cloud computing, virtualization, and slice-as-a-service.
\end{abstract}

Resumo. A virtualização e o fatiamento de recursos vêm se tornando abordagens chaves para automatizar, tornar mais eficiente e econômico o processo de configuração de nuvens, habilitando a oferta de serviços ágeis e dinâmicos. Este artigo apresenta o Centro de Dados Itinerante (Itinerant Data Center - IDC), com o objetivo de levar serviços essenciais para regiões sem ou com pouca infraestrutura, utilizando a plataforma NECOS, para fornecer uma solução sustentável, de baixo custo e com pouco consumo de energia, que utiliza computação em nuvem, virtualização e fatiamento como serviço.

\section{Introdução}

A maioria dos excluídos digitais vivem em zonas rurais ou regiões de ilhas de países em desenvolvimento, como nas ilhas amazônicas que apresentam uma população de baixa renda, regiões esparsamente povoadas, dependência da malha hidroviária e a falta de infraestrutura de tecnologia da informação. Tais fatores contribuem para tornar o planeta um lugar "desconectado". Para sanar este problema, muitos projetos têm sido desenvolvidos: Projeto Amazônia Conectada [Conectada 2015], balões e drones para o acesso à Internet [Vasconcelos 2017], o CoDPON [Coutinho 2009] e o CELCOM [de Castro et al. 2018].

Diante deste cenário, a conexão à Internet torna-se um elemento essencial para viabilizar os serviços computacionais baseados em nuvens. Esta tecnologia atualmente é imprescindível para os serviços que utilizamos no nosso cotidiano, porém, a computação em nuvem pode perder parte de sua eficiência devido ao advento de novas tecnologias, como a Internet das Coisas (Internet of Things) que geram um grande volume de dados (Big Data), que precisam de atendimento prioritário, inclusive para não sobrecarregar as próprias nuvens e os meios de comunicação [Castilho and Kamienski 2018]. Adicionalmente, a computação em nuvem é vista como um modelo que permite o acesso a recursos computacionais configuráveis (como redes, servidores, armazenamento, aplicações 
e serviços) por seus clientes que pagam conforme a capacidade reservada dos recursos, independente da efetiva utilização destes [Marques and Obelheiro 2017].

No entanto, o aumento da latência na computação em nuvem, devido a distância entre a nuvem e os dispositivos finais pode ser um problema para aplicações sensíveis à latência. Visando, entre outros pontos, minimizar a latência dos serviços em nuvem, a computação em névoa surgiu. A névoa (Fog) é alocada no ponto mais conveniente entre o usuário final e a nuvem (Cloud), de modo a otimizar o processamento, armazenamento e transmissão da informação, proporcionando melhoria em todo o processo de comunicação de dados ao deixar a computação mais próximo da borda [Castilho and Kamienski 2018].

Para fornecer uma abstração única dos recursos das nuvens (processamento e armazenamento) e de redes, a plataforma $\operatorname{NECOS}^{1}$ utiliza o conceito de (cloud network slicing) [Silva et al. 2018]. Este conceito ainda apresenta várias definições, mas, no geral, uma fatia da nuvem pode ser definida como uma rede lógica fim a fim (end-to-end - E2E) sob demanda, contando com uma infraestrutura subjacente comum, composta de recursos físicos e/ou virtuais, com controle, gerenciamento e orquestração independentes. Estas fatias devem ser mutuamente isoladas e flexíveis o suficiente para acomodar diferentes necessidades dos inquilinos (tenants), fornecendo um modelo de Fatia como um Serviço (Slice-as-a-Service) e fornecendo a gerência virtual da infraestrutura (Virtual Infrastructure Manager - VIM) sob demanda [Clayman et al. 2018].

Nesse contexto, este artigo implementa a plataforma NECOS com o uso de Computação em Névoa e migração de serviços, para propor o uso de Centro de Dados Itinerante (Itinerant Data Center - IDC) dentro de uma fatia (slice). A essência da proposta é ter uma infraestrutura em um barco, que hospeda microsserviços sob demanda, permitindo a oferta de serviços nas ilhas sem acesso à Internet, ou seja, onde a população é excluída digitalmente. Esses serviços são alocados em fatias na nuvem e a migração/replicação do serviço é realizada no equipamento do IDC. Assim, além de propiciar um ambiente com serviços de redes diversos, será possível atingir maiores públicos levando até essas regiões um ambiente que dê suporte à projetos, como a educação a distância, agendamentos de consultas, atendimentos médicos à distância entre outros.

Além desta seção introdutória, este artigo é composto de mais 5 seções. A Seção 2 apresenta a arquitetura da plataforma NECOS na qual o IDC foi implementado. A Seção 3 descreve os trabalhos relacionados. A Seção 4 apresenta o IDC, seguido de sua aplicação na Seção 5. Enquanto a Seção 6 apresenta as conclusões gerais.

\section{Arquitetura NECOS para Slicing}

A arquitetura completa da plataforma NECOS define os componentes necessários para o aprovisionamento de fatiamento E2E em domínios de federação multi-tecnológicos e de multi-provedores [Silva et al. 2018]. Na Figura 1 nós apresentamos uma versão simplificada da arquitetura destacando os elementos mais relevantes no contexto do IDC.

Para facilitar a compreensão da arquitetura do NECOS, na Figura 1 nós definimos quatro áreas distintas, isto é, a área Tenant em vermelho, a área de Provedor de Recursos em verde, a área do Marketplace em amarelo e o Provedor de Slice em azul.

\footnotetext{
${ }^{1}$ http://www.h2020-necos.eu/
} 


\section{Marketplace}

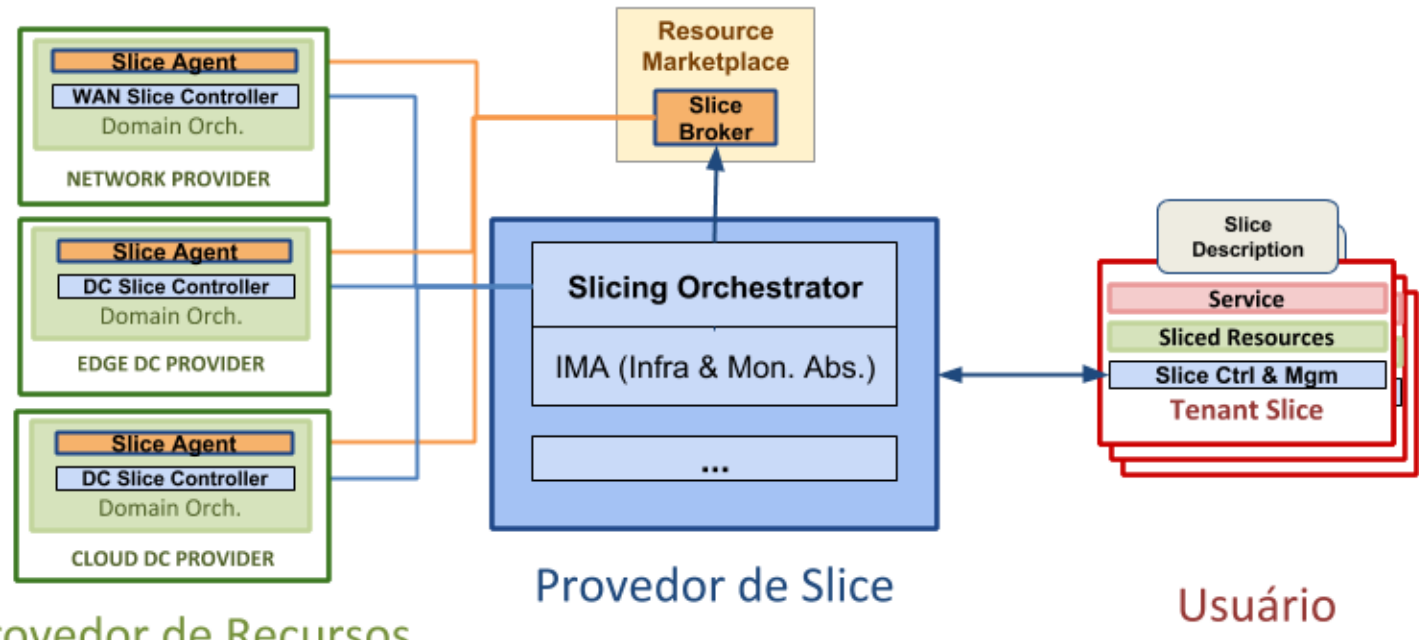

Provedor de Recursos

Figura 1. Visualização abstrata da arquitetura NECOS.

O Slice Orchestrator realiza manipulação de fatias, isto é, requisita ao Marketplace as diferentes partes da fatia que serão incluídas em uma fatia E2E através da realização de uma fase de orquestração inicial. Adicionalmente, ele supervisiona o gerenciamento do ciclo de vida dos serviços que estão sendo executados nas fatias, baseado na informação recuperada do componente $I M A$.

A Abstração de Monitoramento \& Infraestrutura (Infrastructure \& Monitoring Abstraction - IMA) oferece uma interface northbound abstrata que permite ao Slice Orchestrator executar suas funções enquanto os detalhes das partes das fatias permanecem agnósticos, por exemplo, coletando informações sobre topologia de recursos e informações de monitoramento de recursos para cada parte da fatia; monitorando e verificando o status dos elementos virtuais alocados a cada parte da fatia; reunindo Indicadores Chaves de Performance (Key performace Indicators - KPIs) voltados para recursos (como CPU, memória e armazenamento). Para alcançar isso, múltiplos adaptadores traduzem as requisições provenientes da interface northbound para o VIM ou o Gerenciador de Infraestrutura WAN (WAN Infrastracture Manager - WIM). Deste modo, eles escondem as partes das fatias heterogeneamente na interface southbound da IMA.

A descoberta de recursos que compõem uma fatia E2E é designada ao componente Marketplace que é um sistema distribuído responsável por localizar partes de fatia adequadas a partir de um conjunto de domínios de recursos participantes. Como mostrado na Figura 1, o Marketplace consiste em dois sub componentes principais do NECOS, nomeados de Slice Broker e um número de Slice Agents. O Slice Broker descobre as partes das fatias através da interação com um conjunto de Slice Agents hospedados pelos domínios de recursos envolvidos.

\section{Trabalhos Relacionados}

Baseado na tecnologia de Redes Tolerantes a Atraso (Delay Tolerant Networks - DTN) o CoDPON foi proposto com o intuito de viabilizar a integração de programas sociais do governo entre comunidades remotas e grandes centros urbanos, a baixo custo. Para isso 
a rede CoDPON utiliza barcos que se deslocam entre as comunidades com um módulo acoplado que recebe os dados e retransmite ao se aproximar de outros barcos ou a base (cidade). Tal solução não permite que um serviço seja acessado continuamente em um período de tempo especifico, limitando a abrangência da solução [Coutinho 2009].

O projeto SeNDT utiliza redes de sensores tolerantes a atrasos a fim de possibilitar o monitoramento da qualidade da água de lagos e da poluição sonora em rodovias. Com o intuito de solucionar o problema da grande extensão geográfica do lago que foi escolhido para monitoramento, foram utilizadas tecnologias de DTNs. Onde, os sensores foram divididos em regiões e mulas de dados (nós DTN móveis com grande capacidade de armazenamento) trafegam entre essas regiões, garantindo a conectividade da rede. Para o monitoramento da poluição sonora em rodovias foram instaladas unidades de sensoriamento, equipadas com antenas IEEE 802.11, que enviam as informações coletadas mediante uma consulta do operador, que pode permanecer em seu veículo durante a operação. Essa solução mostrou-se bastante vantajosa devido ao baixo custo, robustez e tolerância a atrasos [McDonald et al. 2007].

Através de uma metodologia de comunicação assíncrona, Shivani Harnal e Jasbir Kaur (2016) desenvolveram o DAKNET com o objetivo de conectar aldeias (áreas rurais) aos grandes centros urbanos, através da infraestrutura existente de transporte. Para isso o DAKNET utiliza ônibus ou qualquer outro veículo montado com um equipamento denominado MAP (Ponto de Acesso Móvel) que movimenta-se entre as aldeias, onde são instalados dispositivos denominados FAP (Ponto de Acesso Fixo). A partir do momento em que o veículo se aproxima da aldeia, as informações são baixadas automaticamente do FAP para o MAP e posteriormente serão retransmitidos quando o veículo retorna para a cidade. [Harnal and Kaur 2016]

Uma arquitetura de Névoa foi proposta por Kniess e Ruck com o objetivo de melhorar o desempenho no acesso a páginas web requisitadas através de dispositivos conectados a uma rede sem fio. Nesta arquitetura existe um módulo de consistência que atua como um balanceador de carga formado por um número " $\mathrm{n}$ " de servidores de névoa (por exemplo, um centro de dados em nuvem de pequena escala (Cloudlets)), que têm a função de armazenar as requisições mais frequentes dos usuários. Esse armazenamento é feito através de um ranqueamento das requisições recebidas dos usuários da névoa de forma a construir um mecanismo eficiente de resposta, visto que, os servidores atuam como um cache inteligente evitando acessos desnecessários à nuvem. Assim, é o IDC, que utiliza a Computação em Névoa por apresentar características como a baixa latência, a redução de tráfego e o pré-processamento de dados, fazendo com que a computação na borda minimize o volume de dados que precisa ser armazenado [Kniess and Ruck 2016].

A Tabela 1 apresenta uma comparação das características apresentadas anteriormente nesta sessão, mostrando o diferencial entre os projetos existentes CoDPON, SeNDT, DAKNET e a proposta deste artigo. O IDC se diferencia dos demais devido suas tecnologias utilizadas, que garantem que o serviço seja oferecido em comunidades isoladas com pouca ou nenhuma infraestrutura de comunicação, através de um centro de dados itinerante que se desloca para oferecer serviços de TIC no local em que se encontra, e não apenas oferecer conectividade. Através do IDC é possível realizar serviços de rede solicitados pelo cliente, mesmo este estando desconectado do núcleo da rede, pois sucessivamente, os dados serão sincronizados com o centro de dados principal, enquanto 


\begin{tabular}{|c|c|c|c|c|c|}
\hline Projetos & \multicolumn{5}{|c|}{ Características } \\
\hline & Objetivos & $\begin{array}{l}\text { Tecnologia } \\
\text { utilizada }\end{array}$ & Funcionamento & Conectividade & Vantagens \\
\hline CoDPON & $\begin{array}{l}\text { Integrar programas so- } \\
\text { ciais entre locais remo- } \\
\text { tos e os centros urbanos }\end{array}$ & DTN & $\begin{array}{lr}\text { Os barcos } & \text { se } \\
\text { deslocam } & \text { da } \\
\text { origem até } & \text { o } \\
\text { destino } & \end{array}$ & Necessária & $\begin{array}{l}\text { Conectividade a baixo } \\
\text { custo. }\end{array}$ \\
\hline SeNDT & $\begin{array}{l}\text { Monitoramento da qua- } \\
\text { lidade da água de lagos }\end{array}$ & DTN & $\begin{array}{l}\text { Os sensores } \\
\text { trafegam en- } \\
\text { tre regiões } \\
\text { para garantir a } \\
\text { conectividade }\end{array}$ & Necessária & $\begin{array}{l}\text { Conectividade em am- } \\
\text { bientes agressivos. }\end{array}$ \\
\hline DAKNET & $\begin{array}{l}\text { Conectar áreas rurais } \\
\text { aos grandes centros ur- } \\
\text { banos }\end{array}$ & DTN & $\begin{array}{l}\text { Os veículos se } \\
\text { deslocam da ori- } \\
\text { gem até o des- } \\
\text { tino }\end{array}$ & Necessária & $\begin{array}{l}\text { Conectividade a baixo } \\
\text { custo }\end{array}$ \\
\hline IDC & $\begin{array}{l}\text { Permitir o acesso de } \\
\text { serviços de TIC nas } \\
\text { ilhas sem acesso à In- } \\
\text { ternet }\end{array}$ & Fog & $\begin{array}{l}\text { Um único barco } \\
\text { se desloca da } \\
\text { origem até o } \\
\text { destino }\end{array}$ & $\begin{array}{ll}\text { Não } & \text { ne- } \\
\text { cessária } & \end{array}$ & $\begin{array}{l}\text { Levar o serviço até o } \\
\text { cliente com baixo custo }\end{array}$ \\
\hline
\end{tabular}

Tabela 1. Comparação das propostas.

que, tanto o CoDPON , o SeNDT e o DAKNET através da tecnologia de DTN, precisam garantir uma certa conectividade para a realização de seus serviços, diante disto, o IDC se apresenta como uma solução inovadora, sustentável, de baixo custo e com pouco consumo de energia.

\section{Centro de Dados Itinerante (IDC)}

Este artigo apresenta um Centro de Dados Itinerante de borda (Edge IDC) capaz de promover acesso a diversos serviços de TIC. A ideia principal é ter um centro de dados itinerante que possa hospedar microsserviços sob demanda, permitindo a cobertura de ilhas de serviço sem acesso à Internet, ilhas que normalmente não atingem o núcleo da rede para que possam acessar os serviços. Estes serviços (microsserviços) precisam de uma alocação de fatia, a implantação do serviço ocorre na fatia e a migração/replicação do serviço no equipamento do Edge IDC.

\subsection{Elementos da solução IDC}

O IDC é composto por 3 equipamentos chaves: um computador pequeno ( $D C$-slice-part) que hospeda uma fatia de serviços do centro de dados da nuvem, um roteador sem fio (Net-slice-part) que hospeda uma fatia de serviços que age como provedor de serviços de rede, e um fornecimento de energia. Prevemos, o uso de serviços de vídeo (somente leitura) e serviços de captura de questionário (leitura e escrita).

A Figura 2 mostra a arquitetura do Edge IDC. Em um primeiro momento, o Edge IDC recebe uma fatia do centro de dados da nuvem enquanto está conectado a rede. Em um segundo momento, ao se deslocar para ilhas da região, o Edge IDC, agora desconectado, realiza serviços de redes solicitados sob demanda, e que posteriormente serão sincronizados. 







